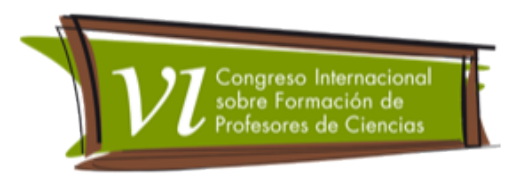

\title{
Conglomerado De Relevancias En El Discurso De Un Niño Llanero Sobre La Naturaleza.
}

Venegas Segura Andrés Arturo', Molina Andrade Adela²

Categoría 2. Trabajo de investigación

\section{Resumen.}

La presente comunicación atiende al discurso sobre la naturaleza de Alfredo por medio de los Conglomerados de Relevancias (Molina 2000, 2012; Venegas 2012a, $2012 b$ ) donde se exponen los diferentes criterios de valor (Naturalista, utilidad, Espacial, Ético-Estético-Emocional) de sus ideas sobre la naturaleza y la jerarquización a los mismos; se muestra la importancia de los criterios naturalista y de utilidad. La comunicación se configura desde la tesis doctoral "Diversidad Cultural, Enseñanza de las Ciencias e Ideas de Naturaleza de Niños y Niñas". Cabe resaltar que su discurso hace referencia a una construcción histórica de los pueblos de la Orinoquia colombiana, donde se reconoce la emergencia de la inclusión de estos saberes en la clase de ciencias y el papel de los mismos en la escuela para una enseñanza no colonialista.

\section{Palabras Clave}

Conglomerados de Relevancias, Ethos llanero, Ideas de Naturaleza,

\section{Introducción y Objetivos.}

El contexto en el cual se llevó la investigación fue la clase de ciencia del cuarto grado en el Colegio Silvino Caro Heredia en el departamento del Vichada, escenario con alta diversidad cultural, donde se encuentran niños y niñas con ascendencia, Sikuani, Piapoco, Llaneros y Colonos. Por lo anterior, se asume la hipótesis que se trata de una clase de ciencias en la cual coexisten valores y creencias culturalmente diferenciadas, lo cual atiende a negociaciones y traspasos de fronteras culturales (Aikenhead, 2001a, 2001b, 1997, 1996; Aikenhead \& Jegede, 1999; Aikenhead \& Olugbemiro, 1999; Candau, 2006; Cobern \& Loving 2001; Cobern, 1996a, 1996b, 1994, 1991; June, 1999; Kawasaki, 1997, 1996; Molina, 2008, 2007a, 2007b, 2004, 2002, 2000; Molina \& Mojica, 2005, 2004; Ogawa, 2000,

\footnotetext{
1 Universidad Distrital Francisco José de Caldas. cDr en Educación. INTERCITEC 2 Universidad Distrital Francisco José de Caldas. Dra en Educación. INTERCITEC
} 
Revista Tecné, Episteme y Didaxis: TED. Año 2014, Número Extraordinario. ISSN Impreso: 0121-3814, ISSN web: 2323-0126

Memorias, Sexto Congreso Internacional sobre Formación de Profesores de Ciencias. 08 al 10 de octubre de 2014, Bogotá

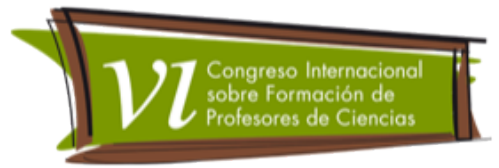

1995, 1989, 1986; Riggs 2004). Así, las ideas, pensamientos, afirmaciones expresadas por los niños y niñas, son negociadas en un contexto escolar con su cultura de formas diversas. El objetivo en este marco es establecer los diferentes criterios de valor acerca de la naturaleza de un niño llanero que es representante de este contexto histórico-cultural.

\section{Referente Conceptual y Metodología}

La metodología de la presente investigación se enmarca en un enfoque cualitativo que permite aproximarse a una interpretación cultural del universo interpretativo del otro. En este sentido no se utilizan categorías predefinidas, sino que por el contrario, se trata de categorías abiertas. En este sentido, se parte de un marco interpretativo de las ideas y de los diálogos a estudiar por medio de los Conglomerados de Relevancias. El Conglomerado de Relevancias se propone con el fin de caracterizar las visiones y perspectivas sobre el mundo natural, resultantes de los intercambios culturales que se presentan en una sociedad caracterizada por su diversidad cultural. Se basa en la idea de valor como decisión de Ricoeur, que permite resolver las opciones de significar presentes en los intercambios entre culturas (Molina, 2000, 2004).

En consonancia, las categorías metodológicas adoptadas se articulan epistemológicamente con el concepto de conglomerado de relevancias (Molina, 2000, 2008) y son tres: (a) idea de narrativa, esto es, la forma como se concebirán las cartas de los niños y las niñas, sus diálogos y dibujos; (b) contenido semántico, los criterios de interpretación de las declaraciones de los niños y las niñas y; (c) contexto cultural.

Su discurso en torno a la idea naturaleza está implicado en sus narraciones, dibujos comentados y diálogos, con base en tres instrumentos (carta al extraterrestre, dibujo sobre la naturaleza y entrevista semi-estructurada). Las interpretaciones de su idea sobre la naturaleza se realiza a partir de los instrumentos mencionados y el relevamiento documental, este último basado en la metodología de análisis de documentos.

\section{Descripción General De Las Jerarquías Del Conglomerado De Elevancias}

La interpretación de las respuestas de los tres instrumentos trabajados con Alfredo muestran varias jerarquías que conforman el Conglomerado de Relevancias de su idea de naturaleza. Éste se configura a partir los criterios Ético-Estético-Emocional (C E-E-E), Naturalista (CN), Espacial (CT) y de Utilidad (CU). Estos se encuentran 
Revista Tecné, Episteme y Didaxis: TED. Año 2014, Número Extraordinario. ISSN Impreso: 0121-3814, ISSN web: 2323-0126

Memorias, Sexto Congreso Internacional sobre Formación de Profesores de Ciencias. 08 al 10 de octubre de 2014, Bogotá

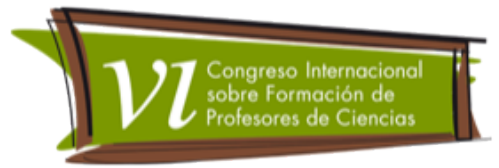

relacionados en diferentes niveles de jerarquía, como se observa en el siguiente gráfico:

Gráfico 1. Jerarquías del Conglomerado de Relevancias para Alfredo

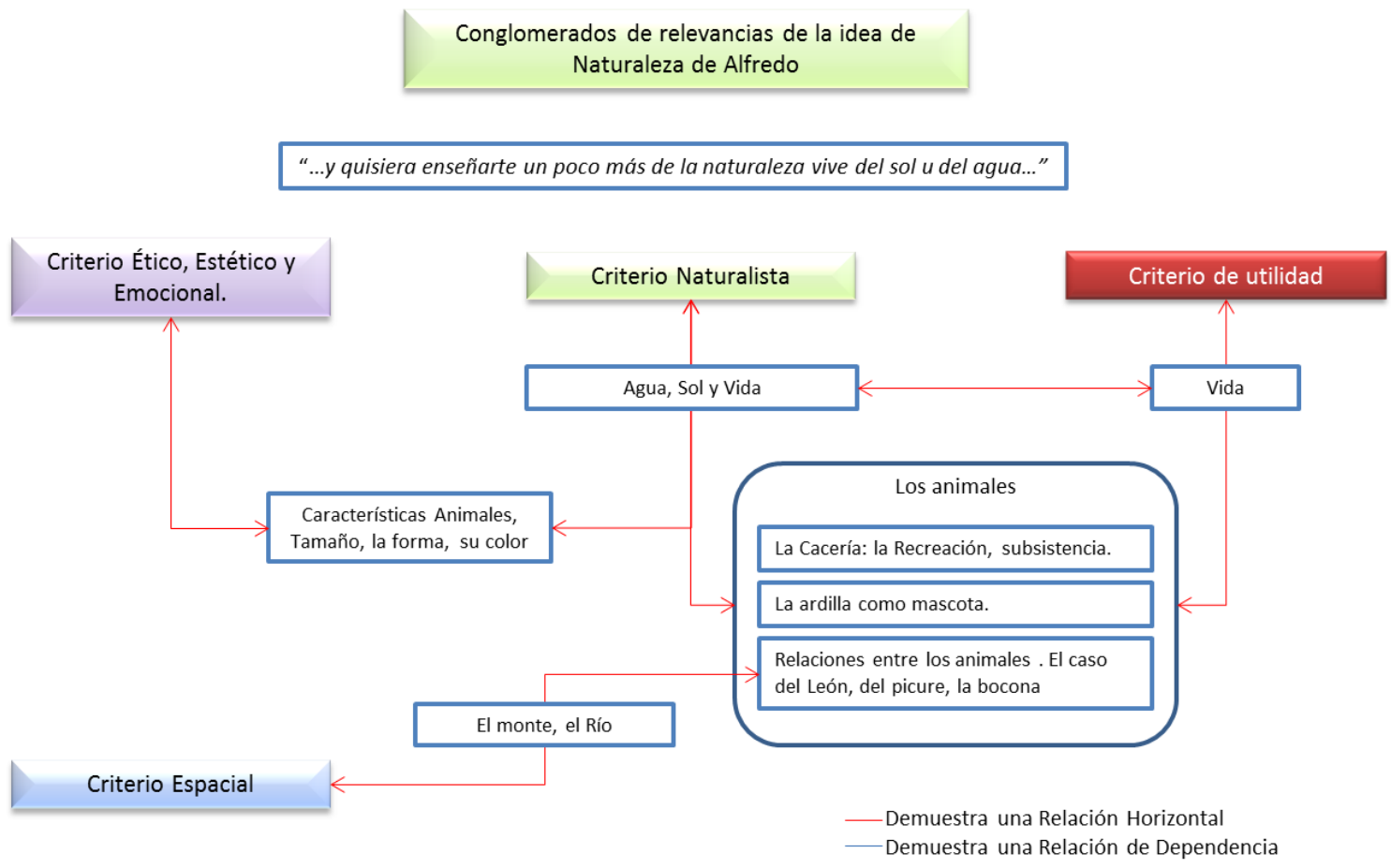

Las Jerarquías indicadas en el Conglomerado de Relevancias implican los criterios Ético-Estético-Emocional (C E-E-E), Naturalista (CN), Espacial (CT), y de Utilidad (CT), sus relaciones se describen a continuación:

- Jerarquía Horizontal uno $(\mathrm{JH} 1<->C N-C U)$ : Configurada entre el criterio naturalista y de utilidad. El conocimiento que Alfredo posee sobre las características de ciertos animales (CN) le permite realizar actividades como de caza y pesca (CU), explica sobre los patrones de caza de los leones que le permiten alimentarse. Se presenta como la naturaleza brinda la vida por medio del oxígeno, aspecto que se exalta en la siguiente afirmación, "La naturaleza es la que da el oxigeno a los seres vivos y a los seres humanos" (Alfredo: Carta).

- Jerarquía Horizontal dos (JH2 <-> CN-C E-E-E): Configurada entre el criterio naturalista y E-E-E. En esta jerarquía se muestra como el conocimiento sobre la fauna presente en el entorno de Alfredo esta expresado por aspectos 
Revista Tecné, Episteme y Didaxis: TED. Año 2014, Número Extraordinario. ISSN Impreso: 0121-3814, ISSN web: 2323-0126

Memorias, Sexto Congreso Internacional sobre Formación de Profesores de Ciencias. 08 al 10 de octubre de 2014, Bogotá

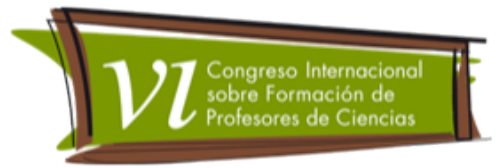

estéticos, es decir, por la forma y apariencia de cada uno de los animales que describe. Al referirse a su deseo (CEm) de enseñar sobre la naturaleza revela múltiples emociones y conocimientos sobre ella.

- Jerarquía horizontal tres (JH3 <-> CU-C E-E-E): Configurada entre el criterio de utilidad y el E-E-E. En esta jerarquía se presenta la cacería como actividad que proporciona alegría, recreación, afianza lazos de reciprocidad entre familiares y amigos. El uso de la naturaleza permite la obtención de recursos alimenticios, en donde se hace presente la regulación en el manejo del entorno (CEt), revela emociones y sentimientos (CEm) con respecto a la naturaleza.

- Jerarquía horizontal cuatro (JH4 <-> CN-CT): Configurada entre el criterio naturalista y el espacial. Los comportamientos de los animales descritos por Alfredo están precedidos por el espacio donde desarrollan su vida, por ejemplo: el Picure obtiene su alimento y vive en el monte; la Bocona o el Bagre encuentra su lugar de vida y alimento en el río, "Alfredo': Esa vive en el agua / Investigador: (...) ¿Qué come la bocona? / 'Alfredo': Pepas (...) Come carne (...) Gusanos, de esos que estábamos juagando ahí en el salón." (Alfredo: Entrevista)

- Jerarquía Horizontal cinco (JH5 <-> CU-CT): Configurada entre el criterio de utilidad y el espacial. En esta jerarquía se encuentra la relación entre la práctica de caza y el conocimiento que Alfredo tiene sobre los lugares que habitan ciertos animales, así, el monte es el lugar de cacería de la lapa y el picure, y el río donde se pesca la bocona.

\section{Conclusiones}

Los criterios (C E-E-E, CN, CU, CT) le permiten a Alfredo constituir su idea de naturaleza donde todos ellos son igualmente importantes. En este sentido, estos criterios facilitan el reconocimiento y compresión de su idea de naturaleza, donde se muestra la diversidad de animales presentes en la naturaleza, sus comportamientos, sus pautas de alimentación, las relaciones entabladas entre el ser humano y ellos, que da paso a formas de pesca, cacería y a la utilización de las flechas y la escopeta; además el uso tanto de flechas permiten observar los patrones de socialización de un sin número de niños y niñas de esta región ya que ellos aprenden su manejo desde muy pequeños y adquieren destrezas particulares frente a su utilización, que les ayudan a sobrevivir en este contexto. Así, la cacería proporciona el desarrollo de conocimientos, destrezas y habilidades que posibilitan la subsistencia y la recreación, donde el juego se configura como fundamental para el aprendizaje, aspectos vinculados al 
Revista Tecné, Episteme y Didaxis: TED. Año 2014, Número Extraordinario. ISSN Impreso: 0121-3814, ISSN web: 2323-0126

Memorias, Sexto Congreso Internacional sobre Formación de Profesores de Ciencias. 08 al 10 de octubre de 2014, Bogotá

conocimiento sobre los animales y sus espacios de vida, a las características que permiten reconocer la diversidad de seres presentes en su contexto: la Orinoquía Colombiana.

Por consiguiente, la idea de naturaleza de Alfredo se configura a través de su relación con el entorno natural, donde la actividad de la cacería cobra importancia en el conocimiento y compresión sobre la naturaleza para posteriormente enseñar sobre ella. La naturaleza se consolida como una entidad que se reproduce con un fin específico: proveer la vida, las condiciones para ésta, el alimento para el ser humano y los animales. La idea de naturaleza de Alfredo y el conocimiento que posee sobre la misma contempla algunos procesos que según él generan la vida de la naturaleza, "la naturaleza vive del sol y del agua..." (Alfredo: Carta). Cabe resaltar que Alfredo ubica a los seres humanos y a los seres vivos en su idea de naturaleza: "La naturaleza es la que da el oxigeno a los seres vivos y a los seres humanos...". Resalta el papel de los animales a través de expresiones como, "...el león el rey de la selva es carnívoro y los tigres también son carnívoros...". Expone la diversidad con respecto a los animales, y son ubicados dentro del mundo, "...Extraterrestres quisiera enseñarte un poco mas de nuestro mundo de los animales son de muchas clase..."

Por otra parte, se hace necesario la inclusión de los saberes ancestrales y tradicionales en el escenario escolar ya que permite integrar otras formas y dinámicas desde la cultura a la clase de ciencias, para evitar enfocar el conocimiento en una dirección, al invisibilizar a las demás culturas. Así, cabe recordar que la incorporación de la ciencia al país, posee atributos de orden colonialista, como los expresados por Nieto (2006), orden fundamentado en la mercantilización de los recursos. Así las ideas expresadas por Alfredo posibilitan su incorporación a la clase de ciencias, en consecuencia también se trata de un acto de reivindicación, visualización y reconocimiento en pro del empoderamiento de las comunidades a través de una enseñanza de las ciencias que conduce a la ruptura de procesos colonialistas y al rescate de la identidad cultural. Retomando a Molina y Mojica (2013), "se requiere una visión crítica de la ciencia asociada con la dominación de una cultura sobre otra, para avanzar en su propósito de lograr el respeto del otro en el campo de la enseñanza de las ciencias" (pág. 42). 
Revista Tecné, Episteme y Didaxis: TED. Año 2014, Número Extraordinario. ISSN Impreso: 0121-3814, ISSN web: 2323-0126

Memorias, Sexto Congreso Internacional sobre Formación de Profesores de Ciencias. 08 al 10 de octubre de 2014, Bogotá

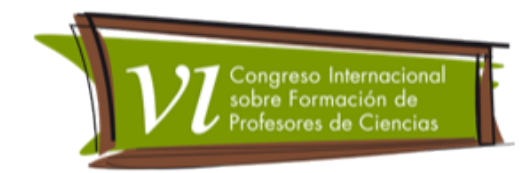

\section{Agradecimientos}

A los niños y niñas del colegio Silvinio Caro Heredia. A las profesoras Claudia Pineda, Blanca Ballesteros, Diana Bautista y Viviana Zorro. Al centro de investigaciones de la Universidad Distrital (CIDC).

\section{Referentes bibliográficos}

Aikenhead, G.S. \& Jegede, O.J. (1999). Cross-cultural science education: A cognitive explanation of a cultural phenomenon. Journal of Research in Science Teaching, 36, pág. 269-287.

Aikenhead, G.S. \& Olugbemiro, J.J. (1999). Cross-Cultural Science Education: A Cognitive Explanation of a Cultural Phenomenon. Journal of Research in Science Teaching, 36 (3), pág. 269-287.

Aikenhead, G.S. (1996). Science education: Border crossing into the subculture of science. Studies in Science Education, 27, pág. 1-52.

Aikenhead, G.S. (1997). Toward a First Nations cross-cultural science and technology curriculum. Science \& Education, 81, pág. 217-238.

Aikenhead, G.S. (2001a). Integrating Western and Aboriginal Sciences: CrossCultural Science Teaching. Research in Science Education, 31 (3), pág. 337355.

Aikenhead, G.S. (2001b). Rekindling Traditions: Cross-Cultural Science \& Technology Units Indigenous Knowledge Conference. Project. http://capes.usask.ca/ccstu.

Candau V.M, Nehme, S. (2006, maio-ago). Conversas com... Sobre a didática e a perspectiva multi/intercultural. Educ. Soc., Campinas, 27, n (95), pág. 471493.

Cobern, W. (1991). World View Theory and Science Education Research. Manhattan-Kansas: NARST.

Cobern, W. (1994a). World View, culture, and science education. Science Education International, 5(4), pág. 5-8. 
Revista Tecné, Episteme y Didaxis: TED. Año 2014, Número Extraordinario. ISSN Impreso: 0121-3814, ISSN web: 2323-0126

Memorias, Sexto Congreso Internacional sobre Formación de Profesores de Ciencias. 08 al 10 de octubre de 2014, Bogotá

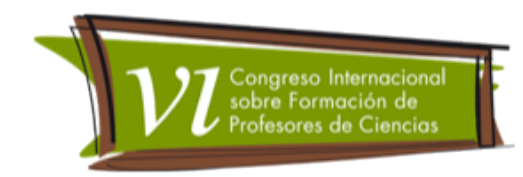

Cobern, W. (1996a). Constructivism and Non-Western Science Education Research. International Journal of Science Education, 4(3), pág. 287-302.

Cobern, W. (1996b). World View, theory and conceptual change in science education. Science Education International, 80(5), pág. 579-610.

Cobern, W., \& Loving, C. (2001). Defining 'Science' in a Multicultural World: Implications for Science Education. Science \& Education, 85, pág. 50-67.

June, G. (1999). World View Analysis of Knowledge in a Rural Village: Implications for Science Education. Science Education, 83 (1), pág. 77-95.

Kawasaki, K. (1996). The concepts of science in Japanese and Western education. Science \& Education, 5, pág. 1-20

Kawasaki, K. (1997). An aspect of science education in Japan within the framework based on structural linguistics. In Ogawa, M. (Ed.), Effects of traditional cosmology on science education pág. 48-62.

Molina, A. (2002). Conglomerado de relevancias y formación científica de niños, niñas y jóvenes. Revista Científica (4), pág. 187-200.

Molina, A. (2000). Conhecimento, Cultura e Escola: Um estudo de suas Interrelações a partir das ideias dos alunos (8-12 anos) sobre os espinhos dos cactos. [Teses doutoral], Doutor em Educação, Faculdade de Educação, Universidade de São Paulo, Brasil.

Molina, A. (2012). Contribuciones metodológicas para el estudio de relaciones entre contexto cultural e ideas sobre la naturaleza de niños y niñas. Bogotá: Universidad Distrital Francisco José de Caldas.

Molina, A., \& Mojica, L. (2013). "Enseñanza como puente entre conocimientos científicos escolares y conocimientos ecológicos tradicionales". MAGIS, Revista Internacional de Investigación en Educación, 6 (12) Edición especial Enseñanza de las ciencias y diversidad cultural, 37-53.

Molina, A., Mojica, L., \& López, D. (2006). Ideas de los niños y niñas sobre la naturaleza: estudio comparado. Revista Científica, 7. pág. 41-62.

Ogawa, M. (1986). Toward a New Rationale of science Education in a Nonwestern Society. European Journal of Science Educaction, 8, pág. 113-119. 
Revista Tecné, Episteme y Didaxis: TED. Año 2014, Número

Extraordinario. ISSN Impreso: 0121-3814, ISSN web: 2323-0126

Memorias, Sexto Congreso Internacional sobre Formación de Profesores de Ciencias. 08 al 10 de octubre de 2014, Bogotá

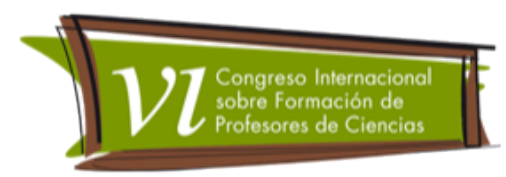

Ogawa, M. (1989a). An introductory Study on traditional Views of nature. The context of Pursuing Rationale for Science Education. Abstract.

Ogawa, M. (1989b). Beyond the tacit framework of "science" and "science education" among science educators. International Journal of Science Education, 11, pág. 247-250.

Ogawa, M. (1995). Science education in a multi-science perspective. In Science \& Education, 79, pág. 583-593.

Ogawa, M. (2000). International joint research on culture, language and gender sensitive science teacher education program. [Unpublished document]. Hiroshima University.

Riggs, E.M. (2004). Components of Geoscience Education For Native American Communities Scence Education. Science Education, 89 (2). pág. 296-313

Venegas S.A.A. (2012 a). La naturaleza, fuente de vida y diversidad que se debe conservar. El discurso de Juan sobre la naturaleza en la clase de ciencias. Revista Nodos Y Nudos V. 4 (33). pág. 62-72.

Venegas S.A.A. (2012 b). Criterios De Análisis en la Interpretación de las Ideas de Naturaleza con los Conglomerado De Relevancias. Revista Científica No. 16 pág. 130-140. 